\title{
Mesoporous Silica Particles Induce Size Dependent Effects on Human Dendritic Cells
}

\section{MATERIALS AND METHODS}

Preparation of mesoporous silica AMS-6 and AMS-8 materials. The general synthesis of AMS- $n$ mesoporous materials has been reported previously. ${ }^{1}$ All reagents were obtained from Sigma-Aldrich and used as received. N-lauroyl Glutamic acid $\left(\mathrm{C}_{12} \mathrm{Glut}\right)$ and $\mathrm{N}$-lauroyl Alanine $\left(\mathrm{C}_{12} \mathrm{Ala}\right)$ surfactants were obtained from Nanologica AB (Uppsala, Sweden). In a typical experiment the surfactant is dissolved at temperatures between room temperature (RT) and $80{ }^{\circ} \mathrm{C}$, in distilled water. After complete dissolution of the surfactant the Co Structure Directing Agent (CSDA) aminopropyl triethoxysilane (APES) is added under stirring at the synthesis temperature followed by the addition of the silica source tetraethyl orthosilicate (TEOS) after a period of time $x$ (where $x$ may vary according to the desired AMS- $n$ mesostructure). For the synthesis of cubic mesocage structure AMS-8; $\mathrm{C}_{12}$ Glut was employed where the final molar composition in the synthesis gel was $\mathrm{C}_{12}$ Glut: $\mathrm{H}_{2} \mathrm{O}$ : APES: TEOS, 1:1830:2.67:11.86 respectively; the dissolution and synthesis temperature was $60^{\circ} \mathrm{C}$ and $x$ was 2 min.

Cylindrical structure AMS-6 was prepared with the $\mathrm{C}_{12} \mathrm{Ala}$ surfactant. In the case of bicontinous cubic AMS-6; the final molar composition in the synthesis gel was $\mathrm{C}_{12}$ Ala: $\mathrm{H}_{2} \mathrm{O}$ : APES: TEOS, 1: 309.1:1.25:6.7, respectively and the dissolution and synthesis temperature was $80^{\circ} \mathrm{C}$.

AMS-8 was maintained at the synthesis temperature for a period of 20 min under stirring, before transferring to an oven at $100{ }^{\circ} \mathrm{C}$ for a further 2 days. The solid samples were then filtered, and dried overnight at $40{ }^{\circ} \mathrm{C}$ under flowing air. AMS-6 was filtered, and dried overnight at $40{ }^{\circ} \mathrm{C}$, after 2 days at the synthesis temperature. 
Samples were calcined in an oven at $550{ }^{\circ} \mathrm{C}$ in a flow of nitrogen and oxygen, in order to remove the surfactant. For extracted samples, 1 gram of as-synthesized material was refluxed at $85{ }^{\circ} \mathrm{C}$ in a $500 \mathrm{~mL}$ mixture of ethanol/ $\mathrm{HCl}(37 \%)$ with ratio $(3: 1)$ for a period of $12 \mathrm{~h}$. The procedure was repeated twice before filtering and drying at RT. In order to increase the monodisperity the final calcined and extracted mesoporous products powders were sonicated for $10 \mathrm{~min}$ at 65 Watts in a Sonic Vibracell ultra sonication apparatus, prior to use.

Characterization of mesoporous nanoparticles. X-ray diffraction (XRD) patterns were recorded on an Xpert Pro Panalytical powder diffractometer equipped with $\mathrm{Cr}$ radiation source $(45 \mathrm{kV}, 40 \mathrm{~mA})$ at the rate of $1.0 \mathrm{deg} / \mathrm{min}$ over the range of $0.8-6.0^{\circ}$. Nitrogen adsorption-desorption isotherms were measured at $-196{ }^{\circ} \mathrm{C}$ on an ASAP2000 Micromeritics Instrument. Calcined mesoporous material was outgassed for a period of 6 $\mathrm{h}$ at $200{ }^{\circ} \mathrm{C}$ and $0.3 \mathrm{kPa}$ pressure. The BET specific surface area was evaluated from the adsorption data in the relative pressure range from 0.05 to 0.3 . The total pore volume was estimated from the amount adsorbed at the relative pressure of 0.97 . Pore size distribution curves were derived using the NLDFT method assuming a spherical or cylindrical (for AMS-6) model of cages. Transmission electron microscopy (TEM) was carried out with a JEOL-3010 microscope, operating at $300 \mathrm{kV}$ (Cs $0.6 \mathrm{~mm}$, resolution $1.7 \AA$ ). Images were recorded using a CCD camera (model Keen View, SIS analysis, size 1024 x 1024, pixel size $23.5 \times 23.5 \mu \mathrm{m}$ ) at $30000-100000 \times$ magnification using low-dose condition.

Scanning electron microscopy (SEMi) images were recorded using a LEO 1550 SEM, equipped with a Schottky field emisson gun. The SEMi was operated at $3 \mathrm{kV}$ and at magnifications of between 20000 and $50000 \mathrm{x}$. The amount of loading of FITC within the cages of mesoporous materials was determined by Thermogravimetric analysis (TGA) performed on a Mettler TGA instrument by heating the samples from 25 to $900{ }^{\circ} \mathrm{C}$ at a heating rate of $10{ }^{\circ} \mathrm{C} / \mathrm{min}$ on an alumina holder under the flow of air at $20 \mathrm{~mL} / \mathrm{min}$.

Fluorescein Isothiocyanate (FITC) labelling of silica particles. Extracted mesoporous nanoparticles of AMS-6 and AMS-8 were used for confocal microscopy analysis since 
the surface of these materials will be funtionalised with propyl amine groups upon extraction, as reported previously. ${ }^{1}$ For the preparation of AMS-n_FITC $500 \mathrm{mg}$ of extracted solid was mixed directly with a solution of FITC (0.0003 moles, SigmaAldrich, Steinheim, Germany) in $20 \mathrm{~mL}$ ethanol. The mixture was stirred for a period of $10 \mathrm{~h}$ at RT in the dark in order to prevent bleaching of the dye. The remaining suspension was filtered and washed repeatedly with ethanol and distilled water in order to dissolve unattached FITC. Finally the sample was dried in an oven overnight at $40{ }^{\circ} \mathrm{C}$.

The loading of FITC on mesoporous AMS-n materials was determined by means of Thermogravimetric analysis (TGA). The figure below shows that for the as-synthesized (not calcined) sample and FITC loaded mesoporous ASM-6 material.

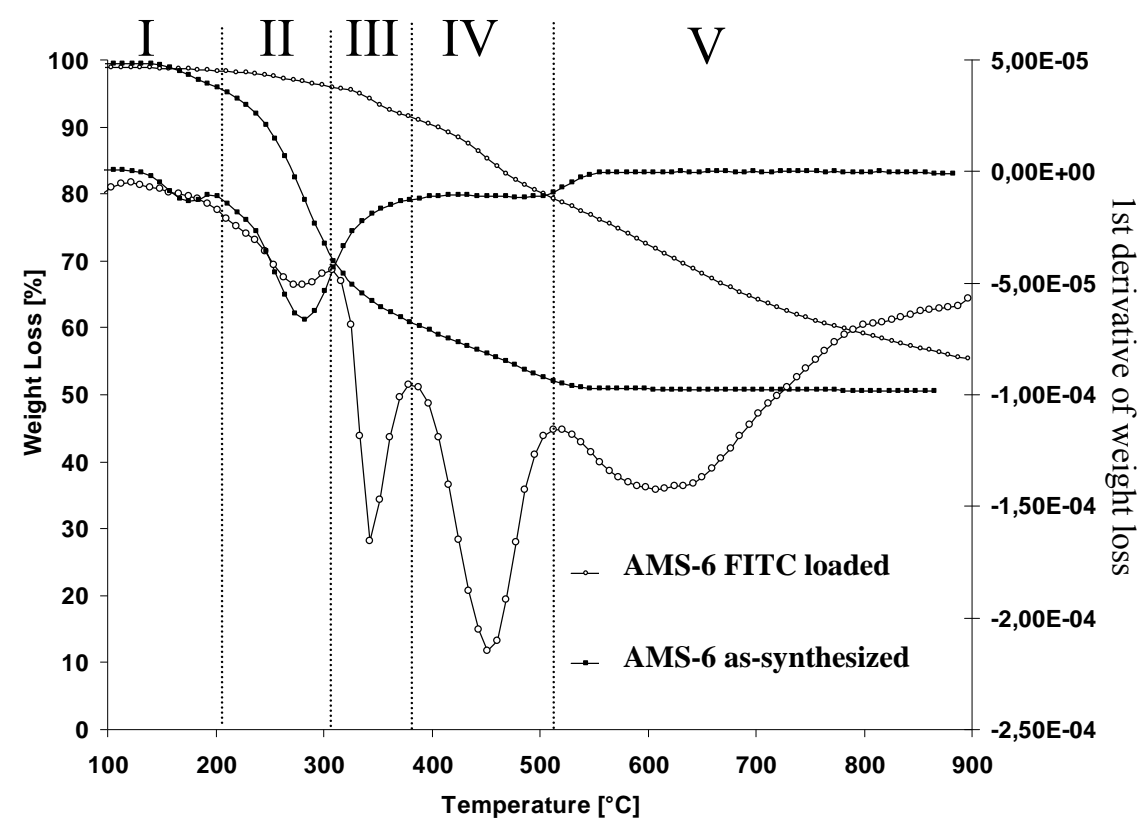

Figure S1. The figure shows a typical TGA curve for loaded and as-synthesized mesoporous AMS-6 particles including weight loss (left y-axis) and the first derivative of the weight loss (right y-axis), both with respect to temperature. The different stages $(I-V)$ of inorganic decomposition have been marked directly in the figure (see text below for details).

The TGA curves for FITC loaded AMS-6 mesoporous material can be interpreted by considering five different weight losses. These correspond to; Stage I (150-200 $\left.{ }^{\circ} \mathrm{C}\right)$ 
consistent with decomposition of external surface propyl-amine functional groups; Stage II which is consistent with the loss of surfactant, and where as much as $3 \%$ of the total weight of the FITC loaded AMS-6 was found to still be anionic surfactant; Stage III $\left(300-400{ }^{\circ} \mathrm{C}\right)$ corresponds to aminopropyl groups covalently bound to the internal mesoporous surfaces; Stage IV can be associated with the decomposition of FITC, the slight higher onset of decomposition in comparison with standard temperatures $\left(350{ }^{\circ} \mathrm{C}\right)$ owing to the electrostatic interaction with surface amine groups; and finally Stage V which is associated with dehydration/condensation of intrawall silanol groups. The loading of FITC is consistent with 13 weight $\%$.

LPS analysis. Each batch of silica particles produced were controlled for lipopolysaccharide (LPS) concentrations by the endpoint chromogenic LAL test method (Limulus Amebocyte Lysate endochrome, Charles River Endosafe, Charleston, SC, USA) according to the manufacturer's instructions. LPS concentrations measured were always below $0.08 \mathrm{ng} / \mathrm{mL}$ which was further reduced to $2 \mathrm{pg} \mathrm{LPS} / \mathrm{mL}$ when diluted in cell cultures.

Generation of monocyte-derived dendritic cells. Human peripheral blood mononuclear cells (PBMC) from healthy blood donors were separated from buffy coats (Karolinska University Hospital Blood Bank, Stockholm, Sweden) by standard gradient centrifugation with Ficoll Paque (Amersham Pharmacia Biotech AB, Uppsala, Sweden). The PBMC were harvested, and washed three times with PBS, followed by resuspension in MACS-buffer $\left(80 \mu 1 / 10^{7}\right.$ cells) containing $0.5 \%$ BSA, 2 mM EDTA in PBS. AntiCD14 microbeads (Miltenyi Biotech, Bergisch Gladbach, Germany) were added according to the manufacturer's instructions. After $30 \mathrm{~min}$ at $4{ }^{\circ} \mathrm{C}$, the $\mathrm{CD} 14^{+}$cells were separated from the solution by autoMACS (Miltenyi Biotec), and analyzed by flow cytometry (FACSCalibur, Becton Dickinson, Franklin Lakes, NJ, USA) to check for $\mathrm{CD}_{1} 4^{+}$cell purity which was always above $96 \%{ }^{2}$ This work was approved by the local ethics committee. 
Immature MDDC were generated mainly as described before ${ }^{3,4}$ by culturing monocytes in RPMI 1640 medium (HyClone, Logan, UT, USA), supplemented with $25 \mu \mathrm{g} / \mathrm{mL}$ gentamicin (Gibco Invitrogen Corporation, Paisley, UK), 2 mM L-glutamine (Gibco Invitrogen Corporation), $100 \mathrm{IU} / \mathrm{ml}$ penicillin (Gibco Invitrogen Corporation), $100 \mu \mathrm{g} / \mathrm{ml}$ streptomycin (Gibco Invitrogen Corporation), $50 \mu \mathrm{M} \beta$-mercaptoethanol (KEBO-lab, Spånga, Sweden), $10 \%$ heat inactivated fetal calf serum (HyClone, South Logan, UT, USA) and with the recombinant cytokines GM-CSF (550 IU/mL), and IL-4 (800 IU/mL), at a density of $4 \times 10^{5}$ cells $/ \mathrm{ml}$, in $37{ }^{\circ} \mathrm{C}$ with a $6 \% \mathrm{CO}_{2}$ atmosphere. Both cytokines were from Biosource International (Camarillo, CA, USA). After 6 days, the cell surface molecules CD1a, CD11c, CD14 and CD83 were analyzed by flow cytometry to confirm a phenotype of immature MDDC with low CD83 expression (always below $8 \%$ ). ${ }^{3}$ The mean viability of the MDDC, as determined by Trypan blue exclusion, was $94 \%$ (range: $91-96 \% ; n=7)$.

Immature MDDC cultured with silica particles. MDDC, at day 6 of culture, were transferred to 6 well plates (Becton Dickinson, San Jose, CA, USA) at a density of $4 \times 10^{5}$ cells $/ \mathrm{mL}$ in $4 \mathrm{~mL}$ of complete culture medium. Silica particles with diameters of $270 \mathrm{~nm}$ $\mathrm{nm}$ and $2.5 \mu \mathrm{m}$, respectively, $(0.5,5$ or $50 \mu \mathrm{g} / \mathrm{mL})$ were incubated with the cells for 24 or $48 \mathrm{~h}$ at $37{ }^{\circ} \mathrm{C}$ or for $3 \mathrm{~h}$ at $4{ }^{\circ} \mathrm{C}$. As controls, cells cultured in medium alone and cells cultured with LPS $(0.1 \mu \mathrm{g} / \mathrm{mL}$; L8274, Escherichia coli, serotype 026-B6, SigmaAldrich) were used.

Flow cytometric analysis. Cells were labeled with fluorescent phycoerythrin (PE) conjugated mouse monoclonal antibodies (mAbs) specific for: CD1a (Coulter Corporation, Hialeah, FL, USA) and CD11c (Becton Dickinson); and with fluorescein isothiocyanate (FITC) conjugated mAbs specific for: CD40, CD80, CD83, CD86 and HLA-ABC, HLA-DR and CD14 (Becton Dickinson) according to the manufacturer's instructions. Control samples were labeled with isotype-matched antibodies conjugated with the same fluorochrome. Fluorescence was measured with a FACSCalibur flow cytometer (Becton Dickinson). Ten thousand viable cells were counted and analyzed by the program CellQuestPro. 
Cytokine assays. Culture supernatants were harvested at $24 \mathrm{~h}$ and $48 \mathrm{~h}$ and kept at -20 ${ }^{\circ} \mathrm{C}$ until analyzed and repeated freezing and thawing was avoided. Production of IL-10 and total IL-12p70 by MDDC was assayed by ELISA kits (MABTECH AB, Nacka, Sweden) according to the manufacturer's protocol. Samples were run in triplicates. Lowest detection limits were $0.5 \mathrm{pg} / \mathrm{mL}$ for IL-10 and $5 \mathrm{pg} / \mathrm{mL}$ for IL-12p70.

Viability analysis. To test for vitality, DC were examined for the degree of apoptosis and necrosis by measuring the binding of Annexin V-fluorescein (Becton Dickinson) and inclusion/exclusion of propidium iodide (PI; Sigma-Aldrich, St. Louis, MO, USA) using a FACSCalibur flow cytometer. $\mathrm{PI}^{-} /$Annexin $\mathrm{V}^{+}$events defined early apoptosis whereas $\mathrm{PI}^{+} /$Annexin $\mathrm{V}^{+}$signals were counted as late apoptosis or secondary necrosis. ${ }^{5}$ Trypan blue (Sigma-Aldrich) staining was used as a complementary method, and which was performed according manufacturer's instructions.

Confocal laser-scanning microscopy (CLSM) analysis. Following incubation for 10 min or $1 \mathrm{~h}$ with FITC-labelled silica particles, MDDC were fixed in $4 \%$ formaldehyde for 15 min. Staining was carried out using anti-HLA-DR mAb (Becton Dickinson) and PI according to the manufacturer's instructions, followed by washing with PBS. A secondary goat anti-mouse mAb labeled with Alexa Fluor 546 (Molecular Probes, Eugene, OR, USA) was used for detection. Slides were mounted with $90 \%$ glycerol. Florescent images were acquired on a CLSM (TCS SP2; Leica Microsystems, Mannheim, Germany) equipped with one argon and two HeNe lasers. FITC was excited with a 488-nm laser line detecting light in the wavelength region of 560-700 nm. Alexa 546 was excited by a 543-nm laser line with detection of light in the region of 560-700 nm. Z-scans were taken with a step width of $0.5 \mu \mathrm{m}$. 
A
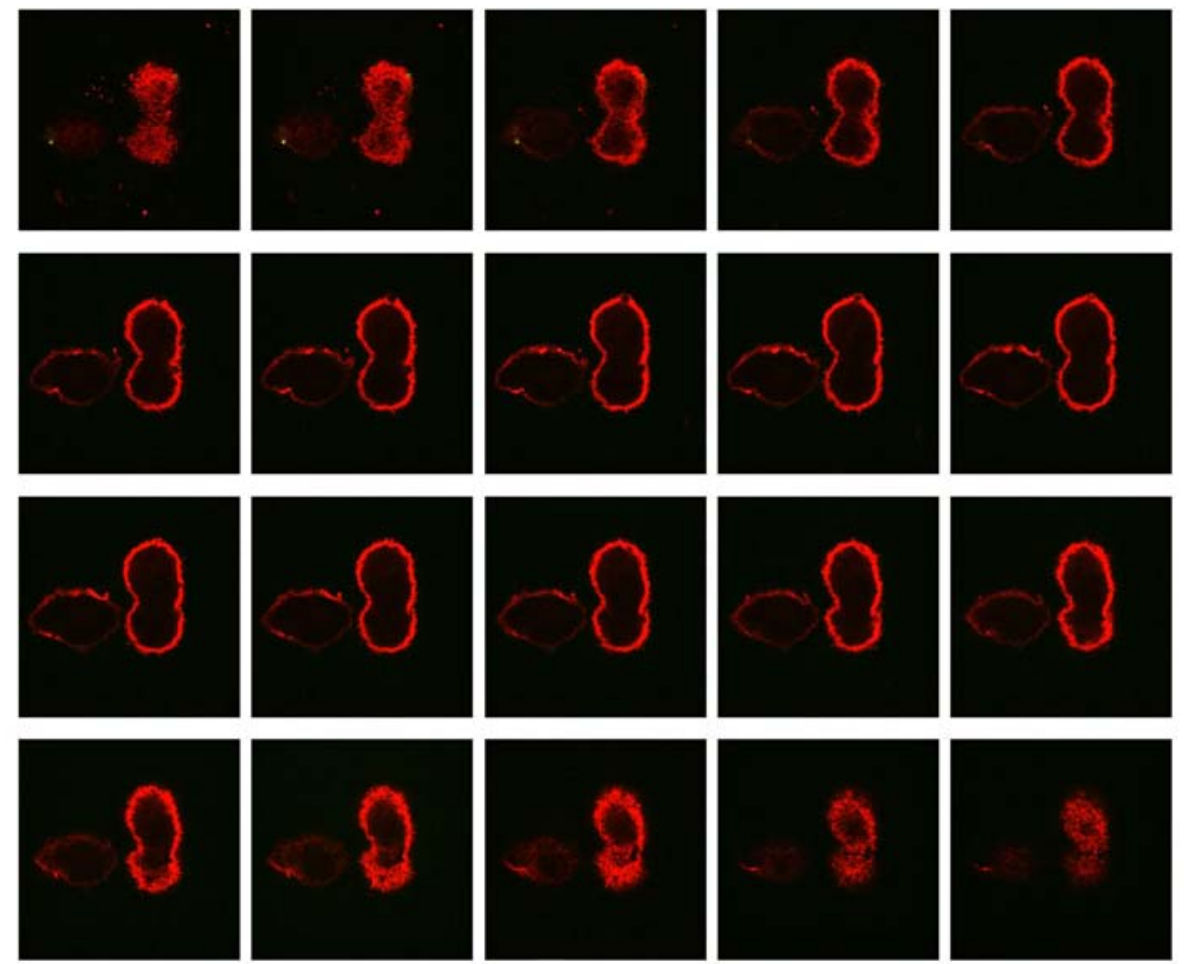

B
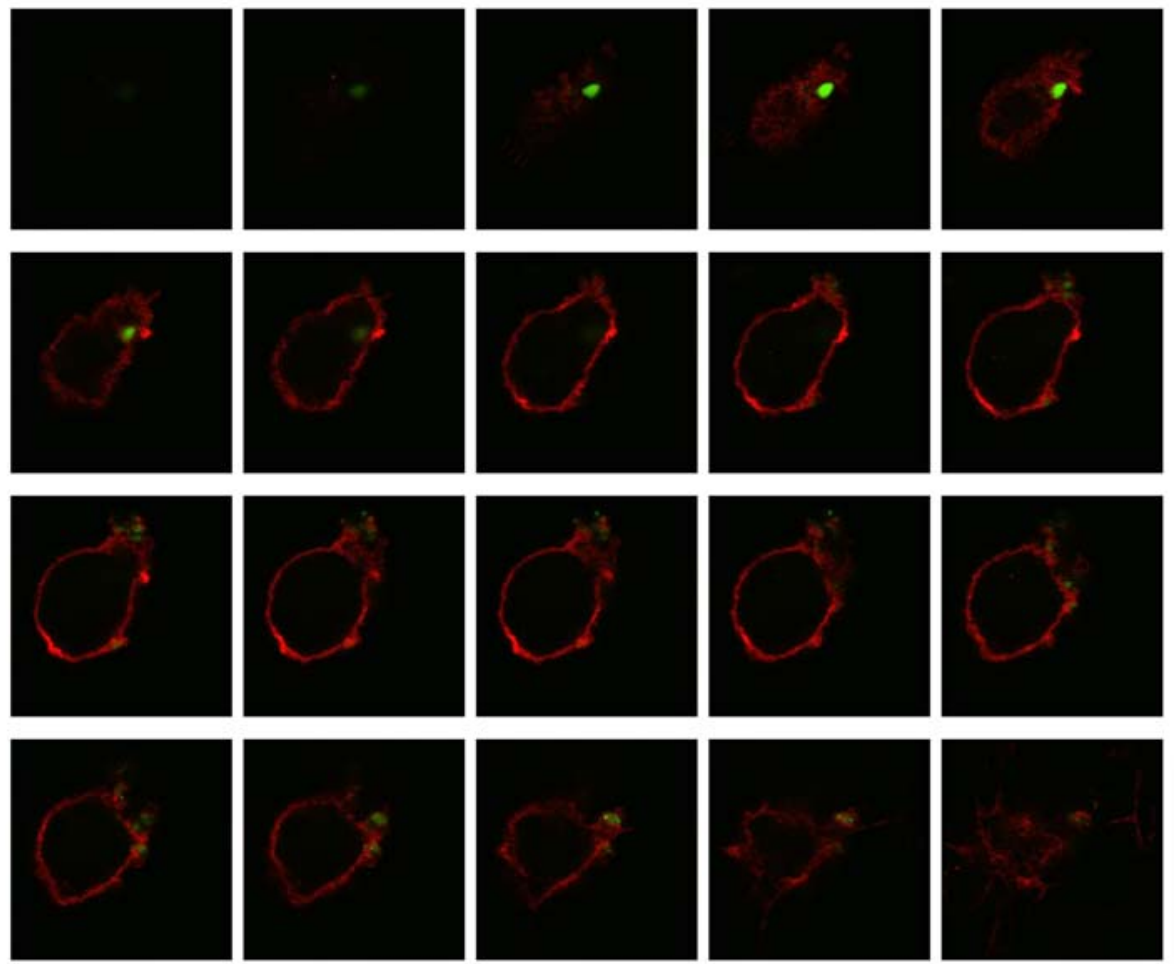

Figure S2. The uptake of the mesoporous silica particles is mediated by an active process. Z-scans show that neither AMS-6 (A) nor AMS-8 (B) were internalized by $M D D C$ following incubations up to $3 \mathrm{~h}$ at $4{ }^{\circ} \mathrm{C}$, although some particles bound to the cell surface. 
Transmission electron microscopy (TEM). MDDC were incubated with silica particles for $1 \mathrm{~h}$ and pelleted. The pellet was fixed in $2 \%$ glutaraldehyde and $0.5 \%$ paraformaldehyde in $0.1 \mathrm{M}$ sodiumcacodylate buffer containing $0.1 \mathrm{M}$ sucrose and $3 \mathrm{mM}$ $\mathrm{CaCl} 2$, pH 7.4 for $24 \mathrm{~h}$ and rinsed in $0.15 \mathrm{M}$ sodiumcacodylate buffer containing $3 \mathrm{mM}$ $\mathrm{CaCl} 2, \mathrm{pH}$ 7.4. The pellets were then postfixed in $2 \%$ osmium tetroxide in $0.07 \mathrm{M}$ sodiumcacodylate buffer containing $1.5 \mathrm{mM} \mathrm{CaCl} 2, \mathrm{pH} 7.4$ at $4{ }^{\circ} \mathrm{C}$ for $2 \mathrm{~h}$, dehydrated in ethanol followed by acetone and embedded in LX-112 (Ladd, Burlington, Vermont, USA). Sections were contrasted with uranyl acetate followed by lead citrate and examined in a Tecnai 10 transmission electron microscope (Fei, The Netherlands) at 80 kV. Digital images were taken by a MegaView III digital camera (Soft Imaging System, GmbH, Münster, Germany).

Statistical analysis. The non-parametric Wilcoxon Signed-Rank Test was used for flow cytometry data and the T-test for dependent samples for viability data. Both tests were run in connection with the STATISTICA software (StatSoft Inc, Tulsa, OK, USA). A pvalue below 0.05 was considered to be statistically significant. When the levels of cytokines were below the detection limit of the ELISA, the value of the detection limit was used in the calculations.

\section{References}

(1) Garcia-Bennett, A. E.; Kupferschmidt, N.; Sakamoto, Y.; Che, S.; Terasaki, O. Angew. Chem. Int. Ed. 2005, 44, 5451-6.

(2) Vallhov, H.; Qin, J.; Johansson, S. M.; Ahlborg, N.; Muhammed, M. A.; Scheynius, A.; Gabrielsson, S. Nano Lett. 2006, 6, 1682-6.

(3) Buentke, E.; Heffler, L. C.; Wallin, R. P. A.; Löfman, C.; Ljunggren, H. G.; Scheynius, A. Clin. Exp. Allergy 2001, 31, 1583-93.

(4) Romani, N.; Gruner, S.; Brang, D.; Kämpgen, E.; Lenz, A.; Trockenbacher, B.; Konwalinka, G.; Fritsch, P. O.; Steinman, R. M.; Schuler, G. J. Exp. Med. 1994, 180, 83-93.

(5) Wang, Y.; Li, X.; Wang, L.; Ding, P.; Zhang, Y.; Han, W.; Ma, D. J. Cell Sci. 2004, $117,1525-32$. 